\title{
Criminologie
}

\section{Les travailleuses du sexe et la vie économique : marginalisation et résistance}

\section{Colette Parent et Chris Bruckert}

Volume 43, numéro 2, automne 2010

Les 50 ans de l'École de criminologie : aperçu de la recherche d'ici et d'ailleurs

URI : https://id.erudit.org/iderudit/1001775ar

DOI : https://doi.org/10.7202/1001775ar

Aller au sommaire du numéro

Éditeur(s)

Les Presses de l’Université de Montréal

ISSN

0316-0041 (imprimé)

1492-1367 (numérique)

Découvrir la revue

Citer cet article

Parent, C. \& Bruckert, C. (2010). Les travailleuses du sexe et la vie économique : marginalisation et résistance. Criminologie, 43(2), 199-217.

https://doi.org/10.7202/1001775ar
Résumé de l'article

Adoptant une approche du travail du sexe comme forme de travail dans le domaine des services, cet article explore comment les travailleuses du sexe, malgré leur autodéfinition comme travailleuses et consommatrices, sont maintenues en marge de la vie économique. Il met en relief les différentes stratégies qui nuisent à leurs activités génératrices de revenu, qu'elles optent ou non pour la rue comme espace de travail. Il explore aussi les résistances et plus particulièrement les stratégies d'évitement qu'elles déploient pour contrer ces tentatives de marginalisation dans le cadre de leur travail et leurs activités de consommation (obtention de carte de crédit, hypothèques, location d'appartement, etc.). En conclusion, il met en évidence le poids de lois sur la trajectoire professionnelle de ces femmes. Les dispositions du Code criminel ont bien sûr un impact majeur sur celle-ci, mais à l'examen, nombre d'autres législations affectent leurs possibilités de se définir comme des travailleuses à part entière. 


\title{
Les travailleuses du sexe et la vie économique : marginalisation et résistance
}

\author{
Colette Parent \\ Professeure titulaire \\ Département de criminologie, Université d'Ottawa \\ coparent@uottawa.ca \\ Chris Bruckert \\ Professeure agrégée \\ Département de criminologie, Université d'Ottawa \\ bruckert@uottawa.ca
}

RÉSUMÉ - Adoptant une approche du travail du sexe comme forme de travail dans le domaine des services, cet article explore comment les travailleuses du sexe, malgré leur autodéfinition comme travailleuses et consommatrices, sont maintenues en marge de la vie économique. Il met en relief les différentes stratégies qui nuisent à leurs activités génératrices de revenu, qu'elles optent ou non pour la rue comme espace de travail. Il explore aussi les résistances et plus particulièrement les stratégies d'évitement qu'elles déploient pour contrer ces tentatives de marginalisation dans le cadre de leur travail et leurs activités de consommation (obtention de carte de crédit, hypothèques, location d'appartement, etc.). En conclusion, il met en évidence le poids de lois sur la trajectoire professionnelle de ces femmes. Les dispositions du Code criminel ont bien sûr un impact majeur sur celle-ci, mais à l'examen, nombre d'autres législations affectent leurs possibilités de se définir comme des travailleuses à part entière.

MOTS-CLÉS - Travail du sexe, vie économique, marginalisation, résistance, stratégies d'évitement, prostitution. 


\section{Introduction}

Depuis quelques décennies, on a assisté à l'émergence d'un discours sur la prostitution comme une forme de travail. Il a d'abord été élaboré au sein des organisations de travailleuses du sexe ${ }^{1}$ qui ont émergé à partir des années 1970 en Occident. Il a donné lieu, entre autres, au Deuxième Congrès international des travailleuses du sexe à Bruxelles en 1986, où l'International Committee for Prostitutes' Rights (ICPR) a adopté une déclaration sur les droits des travailleuses du sexe (Pheterson, 1989). Après avoir énuméré dans un premier temps les nombreuses violations des droits des travailleuses du sexe, le comité a proposé que les travailleuses du sexe aussi bien que les ex-travailleuses se voient accorder les mêmes droits que tous les autres citoyens. Il a également demandé qu'on accorde à la prostitution le statut de profession légitime et que les travailleuses du sexe soient définies comme des citoyennes à part entière.

Cette conception des échanges sexuels contre compensation a aussi été mise en évidence dans les milieux politiques de certains pays dont le New South Wales en Australie et la Nouvelle-Zélande qui, en 1995 et 2003 respectivement, ont décriminalisé les activités de "prostitution» (Corriveau, 2010).

Dans les milieux universitaires, cette approche se présente comme le vis-à-vis de la conception féministe radicale de la «prostitution». Cette dernière définit ces activités comme une forme ultime d'oppression ou d'esclavage. Certains la conçoivent également dans les milieux communautaires et politiques comme une forme de nuisance publique ${ }^{2}$.

Quoi qu'il en soit, on constate quaujourd'hui encore le travail du sexe n'est accepté comme forme de travail qu'en certains milieux. Il s'ensuit que nous n'imaginons pas aisément les travailleuses du sexe comme participant de pleins droits à la vie économique de nos sociétés, bien qu'on puisse documenter leurs activités de travail dans le domaine des services (Parent et Bruckert, 2005), et que ces travailleuses génèrent des revenus qui leur permettent ensuite de participer aux activités de consommation.

1. Dans le cadre de cet article, lorsque nous faisons référence aux travailleuses du sexe qui constituent en nombre la majorité des travailleurs dans ce domaine, le féminin inclut le masculin. Lorsque nous faisons référence spécifiquement aux hommes, nous indiquons le masculin.

2. À ce sujet, voir Shaver (1994). 
Dans le cadre de cet exposé, nous adoptons une approche du travail du sexe comme forme de travail dans le domaine des services et nous explorons comment les travailleuses du sexe, malgré leur autodéfinition comme travailleuses et consommatrices, sont maintenues en marge de la vie économique à travers différentes stratégies qui nuisent à leurs activités de travail et limitent leurs possibilités de consommation. Nous explorons aussi comment elles résistent à cette mise à l'écart afin de demeurer actives dans leur travail et de pouvoir consommer comme les autres citoyens qui ne souffrent pas de discrédit.

\section{Le cadre théorique et les assises empiriques}

Dans nos sociétés capitalistes globalisées, nous constatons que notre statut social renvoie de moins en moins au premier plan à notre appartenance à un groupe, à une religion ou une ethnie. Il s'appuie bien davantage, en termes existentiels, sur notre capacité de gagner des revenus et de dépenser (Moeller, 2006). Cette capacité de gagner des revenus permet aussi de se déplacer dans l'espace et cette mobilité constitue sans aucun doute une des mesures du succès social des individus aujourd'hui (Bauman, 1999).

Mais la capacité de générer des revenus n'est pas un facteur suffisant à lui seul pour nous permettre de définir des activités comme des activités de travail. Dans une publication antérieure, nous avons démontré que le travail du sexe renvoyait à des activités de travail dans le domaine des services (organisation du temps, tablette de services offerts, tarifs, relations avec les collègues, gérants, clients, qualités de base et habiletés à développer, gestion de risques, etc.), mais qu'il n'était pas pour l'essentiel reconnu comme tel. Il constitue une forme de travail non reconnu ou encore en voie d'institutionnalisation (à la lumière des luttes entamées et des processus de reconnaissance en cours ${ }^{3}$ ).

Cette non-reconnaissance, on le comprend, pose le problème du rôle social de la travailleuse du sexe. Or, un rôle social, rappelons-le, implique un cadre d'attentes normatives qui s'imposent aux individus, ou qui tout au moins sert de guide aux comportements (Dahrendorf, 1973). Il oriente les interactions directes entre le client et la travailleuse dans le cadre de la prestation de services, si bien que l'un et l'autre s'appuient sur certaines règles pour structurer leurs échanges. Mais le rôle social

3. À ce sujet, voir entre autres, Parent (2001). 
sert aussi de guide plus général au sein de la société pour apprécier la personne dans son ensemble comme travailleuse et comme citoyenne.

En ce qui concerne la travailleuse du sexe, cette non-reconnaissance a pris une forme exemplaire depuis la fin du $\mathrm{xIx}^{\mathrm{e}}$ siècle en Occident: les travailleuses du sexe constituent depuis lors un groupe de personnes fortement marginalisées (Parent et Coderre, 2000; Parent, 2001). Les lois et la stigmatisation sociale leur bloquent l'accès (inclusion) à de nombreux systèmes sociaux (nous faisons référence ici à la possibilité de conserver la garde de son enfant, de louer un logement, d'obtenir une carte de crédit, une hypothèque, de faire reconnaître son expérience de travailleuse pour être admise dans une institution d'enseignement ou pour obtenir un emploi, de voyager sans problèmes, etc.). On pourrait croire que l'émergence de sociétés globalisées, de sociétés valorisant la croissance individuelle peut éventuellement contribuer à réduire cette marginalisation, ce refus de reconnaissance. Mais dans ces sociétés, le gouvernement des individus, ce que Rose (1999) désigne comme la «conduite des conduites» (the conduct of conduct) est caractérisé par certaines valeurs éthiques qui s'imposent à ceux-ci (Rose, 1999: 331). Aussi, pour beaucoup, les travailleuses du sexe font encore partie des groupes «sans moralité». Elles sont des citoyennes déclassées, jugées incapables de mener leur vie, de gérer de façon responsable les risques de la vie. Il semble bien que dans les sociétés de risques, services sexuels et travail ne peuvent encore et toujours être considérés comme pouvant aller de pair (Parent et Bruckert, 2005).

Par ailleurs, toutes les travailleuses du sexe ne sont pas également exposées et victimes de la marginalisation. En fait, on peut dire que les travailleuses du sexe forment dans les termes de Goffman (1963, 1975 : 57), un groupe discréditable plutôt que discrédité, c'est-à-dire un groupe «dont la différence n'est ni immédiatement apparente ou connue». Que voulons-nous dire par là? Tout simplement qu'elles n'appartiennent pas à un groupe cible identifiable à vue (une personne physiquement handicapée par exemple, une personne appartenant à une minorité visible), mais à un groupe qu'on pourrait en certaines circonstances étiqueter comme différent. En effet, comme la différence postulée entre les citoyens «ordinaires» et les travailleuses du sexe renvoie de façon générale à l'observation de leur présence sur des lieux publics de travail (la rue surtout), où encore à la référence explicite à leurs activités de travail («dans quel domaine travaillez-vous?»), ou à la connaissance préalable de leur domaine de travail, elle n'est généralement reconnais- 
sable que par leur présence en certains lieux ou à travers l'information qu'elles livrent ou qui peut circuler à leur égard. Or étant donné que le travail du sexe de rue expose les travailleuses au regard public, il s'ensuit qu'elles sont plus aisément discréditées que les travailleuses qui choisissent des lieux protégés ${ }^{4}$. Par ailleurs, tant que leur statut social n'est pas connu, les travailleuses du sexe font face à la gestion de l'information en ce qui concerne leur travail. Elles doivent décider à qui la dévoiler, quand, dans quelles circonstances. Elles doivent aussi mettre en place des mécanismes pour contrôler la divulgation et la dissémination de l'information. Lorsque la nouvelle s'ébruite, elles doivent gérer les tensions et les problèmes éventuels qui émergent sur le plan des rapports sociaux et la marginalisation qui peut s'ensuivre.

Or la résistance est, dans les termes mêmes de Foucault (1976), l'autre terme des relations de pouvoir, son irréductible vis-à-vis. Plurielles, ces résistances varient, se concentrent ou s'éparpillent, se fortifient, se transforment ou se dissolvent. Les travailleuses du sexe, comme les autres citoyens, ont la possibilité de résister, de dire non aux mécanismes de marginalisation mis en place.

Bien sûr, elles peuvent le faire publiquement à travers des discours qui définissent le travail du sexe comme une forme de travail et qui demandent la décriminalisation des activités reliées au travail du sexe. Les regroupements de travailleuses du sexe qui se sont multipliés avec plus ou moins de succès depuis les années 1980 en font état ${ }^{5}$. Mais elles peuvent aussi résister individuellement, que leurs actions aient ou non un impact, qu'elles provoquent ou non des mouvements dans le jeu des relations de pouvoir. Ce refus personnel peut prendre la forme de gestes de défiance accompagnés d'une articulation discursive qui met en cause les normes imposées. Des travailleuses du sexe affirment, entre autres, que leur travail remet en question les rapports sociaux de sexe, que les travailleuses du sexe sont des femmes libérées ${ }^{6}$.

La résistance individuelle peut également prendre la forme d'actions dont l'objectif est tout simplement de protéger un espace de travail et

4. Rappelons qu'au xix $x^{\mathrm{e}}$ siècle, les «prostituées» se fardaient, portaient des tenues voyantes et adoptaient des gestes, des attitudes qui attiraient l'attention du public qui circulait. Aujourd'hui, les tenues des travailleuses du sexe de rue se font beaucoup plus discrètes. Voir à ce sujet Parent et Coderre (2000).

5. À ce sujet, voir, entre autres, Matthieu (2001) pour la France, Brock (1998) pour le Canada et Mensah (2010) pour la dimension internationale du phénomène.

6. À ce sujet, voir, par exemple, Bell (1987). 
d'aménager pour soi une vie sociale et économique la plus «normale» possible.

Dans le cadre de cet article, nous examinerons certains mécanismes qui ont pour effet de maintenir les travailleuses du sexe en périphérie de la vie économique aussi bien que les tactiques qu'elles emploient pour résister à leur marginalisation et pour se donner des conditions de vie qui s'apparentent à celles des citoyens qui jouissent de leurs pleins droits. Nous ferons d'abord état des lois pénales adoptées au Canada depuis les années 1980 pour reléguer dans l'ombre les travailleuses du sexe. Nous aborderons ensuite les expériences des travailleuses du sexe de rue, des travailleuses dans les établissements érotiques aussi bien que celles des escortes qui tentent de participer à la vie économique en tant que travailleuses et consommatrices.

Cette présentation prend appui sur trois recherches empiriques que nous avons menées entre les années 2000 et 20097. Nous faisons référence tour à tour aux données tirées: 1) d'une recherche sur le travail du sexe dans les établissement érotiques (appartements, salons de massage) au cours de laquelle nous avons interviewé 14 travailleuses du sexe (Parent et Bruckert, 2005); 2) d'une étude sur les besoins et services des travailleuses du sexe de rue d'Ottawa-Gatineau dans le cadre de laquelle nous avons mené 19 entrevues (Parent et Bruckert, 2006); 3) d'une recherche sur le travail d'escorte dans le cadre de laquelle nous avons réalisé 20 entrevues avec des femmes escortes et 20 avec des hommes escortes des grandes régions d'Ottawa-Gatineau, de Montréal et de Toronto.

\section{Les lois et la marginalisation des travailleuses du sexe de la vie économique}

Lorsqu'on accepte d'observer la «prostitution» comme forme de travail, l'insertion des travailleuses du sexe dans la vie économique de nos sociétés devient évidente. Elles offrent différents types de services sexuels rémunérés, font de bons profits et peuvent consommer comme

7. La première recherche que nous avons menée sur le travail du sexe dans les établissements érotiques a été subventionnée par la Commission du droit du Canada (Parent et Bruckert, 2005); la deuxième, sur les besoins et services des travailleuses du sexe de rue, a été subventionnée par Condition féminine Canada (Parent et Bruckert, 2006); la troisième, sur le travail d'escorte, a été subventionnée par le Conseil de recherches en sciences humaines du Canada (2004-2008). 
tous les autres citoyens. Mais au Canada comme dans nombre de pays occidentaux, cette contribution n'est pas reconnue. En effet, les lois canadiennes n'interdisent pas la prestation de services sexuels contre compensation, mais interdisent différentes pratiques qui lui sont intrinsèquement reliées. Les travailleuses du sexe ne peuvent communiquer sur la voie publique pour fins de prostitution, inciter à la prostitution, vivre des fruits de la prostitution, tenir ou se trouver dans des maisons de débauche. En somme, les lois pénales contribuent largement à discréditer les travailleuses du sexe, entre autres, au sein de la vie économique.

Ce discrédit affecte les espaces de travail des travailleuses du sexe. Les travailleuses du sexe de rue en particulier risquent de se heurter aux stratégies spatiales mises en place par les différents paliers de gouvernement, les forces de l'ordre et les groupes de résidents qui protestent contre leur présence dans leur quartier (Hubbard et Sanders, 2003).

À la fin des années 1970, au Canada, on a assisté à une levée de boucliers devant la présence publique des travailleuses du sexe. Devant les plaintes des forces de l'ordre et des résidents de certains quartiers des grandes villes canadiennes comme Montréal, Ottawa et Vancouver, le gouvernement fédéral a adopté en 1985 l'article 213 du Code criminel sur la communication pour fins de prostitution sur la voie publique ${ }^{8}$. En vertu de cette loi, l'automobile constitue un espace public et le client, aussi bien que la travailleuse du sexe, peut faire l'objet d'une accusation. Depuis cette époque, le travail du sexe de rue peut donc être surveillé de façon plus serrée par les forces policières. D'après une évaluation menée par le ministère fédéral de la Justice cinq années après son entrée en vigueur, cette loi aurait principalement eu comme effet de déplacer les activités de prostitution d'un lieu vers l'autre (Gemme et Payment, 1992). Au demeurant, les conflits entre les résidents et les travailleuses du sexe de rue n'ont pas disparu. Encore aujourd'hui, des associations de résidents (à Montréal et à Ottawa, par exemple) se mobilisent pour

8. 213 (1) «Est coupable d'une infraction punissable sur déclaration de culpabilité par procédure sommaire quiconque, dans un endroit soit public soit situé à la vue du public et dans le but de se livrer à la prostitution ou de retenir les services sexuels d'une personne qui s'y livre: $a$ ) soit arrête ou tente d'arrêter un véhicule à moteur; $b$ ) soit gêne la circulation des piétons ou des véhicules, ou l'entrée ou la sortie d'un lieu contigu à cet endroit; c) soit arrête ou tente d'arrêter une personne ou, de quelque manière que ce soit, communique ou tente de communiquer avec elle.» 
dénoncer le «désordre public» associé aux activités de travail du sexe dans leur quartier et les policiers organisent des blitz pour chasser les travailleuses du sexe de certaines rues.

À l'évidence, les différentes initiatives pour chasser les travailleuses du sexe de rue de leurs lieux de travail se sont heurtées et se heurtent toujours à la résistance des travailleuses. Certaines travailleuses choisissent d'emblée de travailler dans des établissements, que ce soit des salons de massage, des appartements ou des agences de services érotiques. D'autres se déplacent d'un hôtel à l'autre. Elles demeurent donc davantage sous couvert, mais comme indiqué plus haut, elles doivent prendre des mesures pour éviter le discrédit. Comme toutes les travailleuses du sexe, elles doivent également mettre en place des mécanismes pour s'inscrire comme des citoyennes solvables et respectables dans le circuit économique.

\section{Des pratiques de marginalisation}

Les policiers chargés de l'application des lois jouent également un rôle important dans la marginalisation économique des travailleuses du sexe. Ils utilisent d'abord et avant tout la loi sur la communication, qui leur permet d'arrêter une travailleuse du sexe au moindre signe de travail ou de mouvement qui pourrait être interprété comme une forme de sollicitation. D'ailleurs, à l'échelle du pays, les accusations relatives à la prostitution ont augmenté de façon très significative depuis 1986; en 1995, 92\% de ces accusations visaient la communication sur la place publique pour fins de prostitution (Duchesne, 1997). Ils peuvent aussi les arrêter en vertu d'autres chefs d'accusation comme flâner sur la place publique ou encore faire appel à certains règlements municipaux. À Montréal, par exemple, les policiers donnaient des contraventions contre les travailleuses pour avoir arpenté la rue sans respecter les règlements de circulation, pour avoir craché sur la chaussée, etc. Une travailleuse du sexe de rue avait ainsi accumulé 8000 \$ d'amende en une année (Réseau juridique canadien VIH/SIDA, 2005 : 23). À la fin des années 1990, des travailleuses, appuyées par l'organisme Stella, ont décidé de contester ces contraventions au motif qu'elles avaient été émises de façon discriminatoire envers les travailleuses du sexe de rue, violant leurs droits fondamentaux protégés par la Charte canadienne des droits et libertés. Lors d'une audience à la Cour municipale le 31 octobre 2001, 
le procureur de la Couronne a annulé toutes les contraventions et mis fin à la procédure judiciaire (ConStellation, avril 2005 : 86).

Aujourd'hui, dans plusieurs villes canadiennes, dont Montréal et Ottawa, les travailleuses du sexe de rue arrêtées par la police peuvent se voir imposer des conditions de mise en liberté avant de comparaître devant un juge, dont un couvre-feu et l'interdiction de se trouver dans un quadrilatère spécifique (ConStellation, hiver 2005: 30; Power, 2010). Ces conditions que le juge peut aussi imposer dans le cadre d'une ordonnance de probation ont de lourdes conséquences pour ces citoyennes, qui se voient interdire l'accès à leur quartier de travail et souvent n'ont plus la possibilité de joindre leurs clients.

Aussi, compte tenu des lois et de leur application, la plupart des travailleuses du sexe de rue ont un casier judiciaire, ce qui a de lourdes conséquences sur leurs capacités d'être reconnues comme des travailleuses à part entière et de modifier leur trajectoire de travail pour s'insérer sur le marché du travail régulier. Notre recherche sur les besoins et services pour les travailleuses du sexe de rue de la région d'OttawaGatineau nous a d'ailleurs permis de constater que 18 des 19 travailleuses interviewées avaient un casier judiciaire.

Les policiers peuvent également faire pression quotidiennement sur les travailleuses du sexe pour qu'elles quittent la rue, pour qu'elles renoncent à ce lieu de travail. Les travailleuses en ont fait largement état. Brigitte par exemple signale que les policiers essaient toujours de la prendre en faute lorsqu'elle négocie ses services avec un client et certains se font même passer pour des clients. Une fois connue, la travailleuse devient la cible du harcèlement policier dès qu'elle apparaît dans la rue, là où elle recrute sa clientèle. Brigitte précise: «Bien souvent à cause que je suis connue par la police, ils me harcèlent: "Débarque du coin! Si on revient puis on te trouve sur le coin, on te ramasse", pour n'importe quoi là, flâner sur la place publique.» Julie rapporte le même problème. Comme les policiers la connaissent, ils s'adressent à elle et lui conseillent de rentrer à la maison parce qu'ils n'ont pas l'intention de la laisser tranquille et affirment qu'elle ne fera pas d'argent. Selon Jackie, ce sont les policiers qui rendent le travail stressant: «Ils ne te laissent pas travailler, ils te suivent jusqu'à ce que tu en aies assez et décides de rentrer à la maison", déplore-t-elle. La pression peut être très lourde lorsque les policiers maintiennent cette tactique pendant une période de temps assez prolongée. 
À cela, il faut ajouter la violence verbale et physique de certains policiers. Dans notre recherche sur le travail du sexe de rue, neuf travailleuses en font état. Une des travailleuses affirme même qu'elle ne se sent pas tant menacée par les clients que par les policiers qui la traitent très grossièrement.

Aussi les travailleuses estiment que le travail policier nuit à leur travail. Plus encore, les agents ne leur offrent pas la même protection qu'aux autres citoyens. Naomi rapporte qu'un individu violait des travailleuses du sexe dans un quartier d'Ottawa sans que les policiers se donnent la peine d'enquêter. Il a fallu 11 victimes, nous rapporte-t-elle, avant que les autorités n'assignent un policier enquêteur pour investiguer le problème. Daphnée, quant à elle, a constaté combien les droits des travailleuses du sexe pouvaient être bafoués par la police. Victime de la violence d'un client, c'est elle, et non ce dernier, qui a été arrêtée par la police parce qu'elle leur a dit qu'elle était travailleuse du sexe de rue. Les vêtements déchirés, pleine d'ecchymoses et de coupures, ils l'ont emmenée au poste de police.

Les résidents de quartier peuvent aussi nuire au travail des femmes. Dans notre recherche sur les besoins et services des travailleuses du sexe de rue dans la région d'Ottawa-Gatineau (Parent et Bruckert, 2006), quatre travailleuses font état de protestations de certains résidents. On retrouve des personnes qui réagissent en interpellant la travailleuse, l'insultant et l'enjoignant à quitter les lieux. D'autres lancent des objets ou encore se font passer pour des clients et une fois dans la voiture s'en prennent à la travailleuse, tentent de lui infliger une raclée. Certains ciblent des clients et les menacent d'appeler la police. Il y a aussi des résidents qui se regroupent et forment une vigie pour chasser les travailleuses du secteur. Naomi rapporte que dans deux quartiers d'Ottawa (Vanier et Gladstone), on affichait des pancartes indiquant «No johns or hookers we're watching you ${ }^{9}$. Des citoyens sortaient de leur logement pour prendre en note le numéro de plaque d'immatriculation de clients au moment où la travailleuse du sexe entrait dans la voiture. D'autres poursuivaient les travailleuses du sexe avec leur chien afin qu'elles quittent la rue.

Devant ces pressions incessantes, plusieurs travailleuses du sexe de rue choisissent d'abandonner ce lieu de travail et se tournent vers d'autres formes de travail du sexe. Mais celles qui choisissent de tra-

9. «Pas de clients ou de putes, nous vous surveillons» (Notre traduction). 
vailler dans des salons de massage ou chez elles peuvent tout de même être arrêtées pour tenir ou se trouver dans une maison de débauche et perdre encore une fois leur lieu de travail ${ }^{10}$. Leur travail se trouve en permanence menacé. D’abord, bien sûr, des policiers attirés par une publicité jugée trop voyante peuvent intervenir. Ensuite, des résidents témoins de va-et-vient qu'ils jugent suspects ou dérangeants peuvent porter plainte. Enfin, un client insatisfait des services, ou un pseudoclient qui cherche à exploiter les travailleuses, peut à son tour menacer de les dénoncer à la police.

Même si les travailleuses du sexe qui travaillent dans des établissements sont moins menacées au quotidien de perdre leur espace de travail que les travailleuses du sexe de rue, elles n'en sont pas moins vulnérables aux initiatives de la justice.

\section{Des stratégies de résistance}

Les travailleuses, nos recherches le démontrent bien, résistent aux menaces qui pèsent sur leurs activités de travail et au discrédit qui peut en découler et qui contribue à les maintenir en marge de la vie économique citoyenne. Notons ici que compte tenu des contraintes juridiques qui circonscrivent le travail de ces femmes et le discrédit qui les menace, les stratégies de résistance possibles sont, pour beaucoup, des stratégies d'évitement.

D'abord, les travailleuses du sexe de rue mettent généralement en place ce type de stratégies face aux forces policières. Elles peuvent, par exemple, cibler une période de la journée ou encore une journée où, selon leurs expériences, les policiers patrouillent moins. Ainsi, lorsque Emma a commencé à travailler, elle préférait arpenter la chaussée durant la journée, de 10 heures à 17 heures. Naomi signale aussi que la police patrouille peu le jour mais est très présente le soir, surtout les vendredis. Jackie, de son côté, évalue la situation au quotidien. Lorsqu'elle va faire ses courses durant la journée, elle observe la circulation et lorsqu'elle

10. En effet, selon l'article 210 du Code criminel canadien: «(1) Est coupable d'un acte criminel et passible d'un emprisonnement maximal de deux ans quiconque tient une maison de débauche. (2) Est coupable d'une infraction punissable sur déclaration de culpabilité par procédure sommaire quiconque, selon le cas: a) habite une maison de débauche; $b$ ) est trouvé, sans excuse légitime, dans une maison de débauche; $c$ ) en qualité de propriétaire, locateur, occupant, locataire, agent ou ayant autrement la charge ou le contrôle d'un local, permet sciemment que ce local ou une partie du local soit loué ou employé aux fins de maison de débauche.» 
voit passer deux voitures de police en peu de temps, elle se dit que les policiers planifient quelque chose et adapte sa journée de travail en fonction de cette donnée (elle modifie son horaire, par exemple). Les travailleuses peuvent aussi changer fréquemment de lieu de travail pour éviter d'attirer l'attention de la police. Jackie constate que les policiers ont l'habitude de cibler un secteur au détriment des autres. Alors, affirme-t-elle, les travailleuses se déplacent d'un quartier à l'autre, ou encore d'un côté de la rivière Outaouais à l'autre pour échapper à leur surveillance. Dans plusieurs villes, on a noté que les travailleuses du sexe s'étaient déplacées et travaillaient maintenant dans des rues obscures, loin du regard des policiers et des résidents (Lowman, 2000: 22). Michelle, quant à elle, a mis au point une stratégie de fuite. Elle se déplace à bicyclette pour recruter ses clients et peut donc disparaitre rapidement à l'arrivée de la police.

Dans la rue, certaines travailleuses recourent à des stratégies pour éviter d'attirer l'attention des policiers et des résidents. Candy, par exemple, a choisi de déambuler lentement plutôt que de faire du surplace et attirer l'attention des résidents. Elle évite aussi les rues où il y a des enfants. Tracy se fait discrète: elle ne porte pas de vêtements provocants associés traditionnellement à la travailleuse du sexe de rue et travaille en jeans. Qui plus est, elle arpente la rue près d'un arrêt d'autobus où, compte tenu du mouvement régulier des piétons, elle passe inaperçue plus facilement.

Si ce type de stratégies peut leur permettre d'éviter certaines confrontations avec des résidents, celles-ci sont parfois inévitables. Dans un premier temps, même lorsque ces résidents les invectivent, certaines travailleuses décident d'ignorer leurs commentaires. Mais s'ils se mettent à crier, la réplique peut suivre. Tikaboo, par exemple, préfère répondre poliment aux remarques des citoyens, mais s'ils deviennent agressifs, elle riposte. Par ailleurs, Wendy qui, plus jeune, réagissait par la violence aux attaques homophobes dont elle faisait l'objet, choisit maintenant de s'éloigner et de chercher de l'aide lorsque nécessaire.

Ces stratégies de résistance n'ont pas toujours le succès escompté, en particulier lorsque les policiers patrouillent systématiquement dans plusieurs secteurs et que les résidents s'unissent pour chasser les travailleuses. Dans ces circonstances, certaines travailleuses évitent d'arpenter trop souvent la rue et ciblent surtout une clientèle régulière: elles peuvent même rencontrer certains clients chez elles. Certaines peuvent 
aussi décider de quitter la rue. Cela peut signifier la fin de leur insertion dans le domaine du travail du sexe ou encore un changement de secteur.

$\mathrm{Au}$ total, on peut constater que les travailleuses du sexe de rue de la région d'Ottawa-Gatineau résistent aux différentes mesures qui visent à les chasser de leur lieu de travail. D'autres recherches menées ailleurs en arrivent aux mêmes conclusions. Mais pour les travailleuses, le prix à payer peut parfois être élevé. Les évaluations de la loi sur la communication dans l'ensemble du pays indiquent que son application a eu des effets délétères sur les travailleuses dans certaines villes, et en particulier dans le Downtown Eastside de Vancouver. Plus récemment, en 2005-2006, le gouvernement fédéral a créé un sous-comité parlementaire pour étudier l'impact de la loi sur la communication. Ce comité a été mis sur pied à la suite des demandes répétées de la députée de Vancouver-Est, où le tristement renommé Robert Pickton aurait, au fil des années, recruté, amené sur sa ferme porcine et assassiné des travailleuses du sexe de rue du Downtown Eastside de Vancouver ${ }^{11}$. Le sous-comité a entendu des témoignages de spécialistes de différentes disciplines et orientations théoriques qui ont soutenu que la loi sur la communication contraignait les travailleuses du sexe à travailler dans des endroits sombres, isolés, loin des services d'aide et de protection. Ces femmes ont aussi tendance à se disperser pour ne pas attirer l'attention, ce qui les prive de l'appui d'autres collègues (qui peuvent échanger des informations sur les clients et prendre en note leur numéro de plaque d'immatriculation). Elles sont alors très vulnérables aux agresseurs qui se font passer pour des clients. Selon Lowman (2000: 19), ces pseudo-clients, ces agresseurs, ne sont pas enclins à rechercher des travailleuses du sexe qui travaillent dans des établissements ou encore pour une agence parce qu'ils craignent d'être identifiés. Les statistiques indiquent que 78,8\% des femmes assassinées en Colombie-Britannique entre 1975 et 1994 étaient des travailleuses du sexe et elles ne travaillaient ni pour des établissements ni pour des agences (Lowman et Fraser, 1995 : s 2). Selon Lowman et Fraser (1995: s 148), la loi sur la communication a très largement contribué à créer un contexte juridique et social qui a rendu faciles les homicides. C'est une loi qui met en danger les travailleuses du sexe les plus désavantagées.

11. Pickton a été reconnu coupable de meurtre au second degré de 6 travailleuses du sexe et mis en accusation pour le meurtre de 20 autres. Il a maintenant épuisé ses possibilités d'appel et la Couronne a décidé de ne pas poursuivre dans les autres dossiers. Voir «Les 20 accusations de meurtre contre Pickton suspendues», La Presse, 5 août 2010. 
Aussi, plusieurs travailleuses du sexe de rue qui se croient en mesure d'affronter les défis du changement choisissent d'abandonner la rue comme lieu de travail. Angela, considérant qu'il y avait trop de policiers et qu'elle risquait fort d'être arrêtée et mise en accusation, a quitté la rue pour travailler dans les messageries roses. D'autres optent d'entrée de jeu pour travailler dans des lieux protégés comme les établissements érotiques. Plusieurs travailleuses que nous avons interviewées ont souligné que ces établissements leur permettaient de garder l'anonymat et d'éviter le discrédit, et deux d'entre elles ont spécifiquement affirmé que ce choix les protégeait mieux des arrestations policières.

Mais ce refuge entre quatre murs ne constitue pas une mesure de protection suffisante. Celles qui travaillent dans des établissements érotiques doivent également mettre en place des mécanismes pour éviter d'attirer l'attention policière. D'abord, l'entreprise ne peut s'afficher d'une manière trop voyante et ne peut communiquer très explicitement les services disponibles. Cette limite se répercute sur le service et éventuellement sur le volume de la clientèle: ou l'on tend vers une publicité assez explicite au risque d'être dénoncé, ou l'on opte pour la prudence au risque de ne pas attirer de clients. Même les informations offertes au téléphone doivent être formulées dans un langage codé qui sera certes compris par un usager régulier de ce type de services, mais pas nécessairement par un néophyte. Ensuite, on cherche à filtrer les clients de manière à éviter les agresseurs qui peuvent blesser les travailleuses, affecter leur capacité de travail. On met donc en place des vérifications d'identité, au téléphone ou sur place, des caméras cachées à l'entrée, des systèmes d'alarme dans les salles, ou encore on observe le client à distance avant de lui indiquer au téléphone la porte d'entrée. Sur place, les travailleuses du sexe n'expliquent les services offerts et ceux qui sont ne le sont pas que lorsqu'elles sont rassurées sur l'identité du client. Dans les premiers moments d'interaction, comme les travailleuses connaissent peu les lois, elles peuvent recourir à des mesures qu'elles estiment, à tort, avoir pour effet d'éviter d'éventuelles accusations. Ainsi, certaines pensent se protéger en demandant au client de se déshabiller en premier, en ne touchant pas à l'argent, en n'offrant pas de services sexuels complets, etc. Qui plus est, des travailleuses croient que le respect de certaines règles, comme l'absence de mineurs ou de consommation de drogues, leur assure l'impunité.

La mise en place de ces nombreuses mesures assure une certaine protection des lieux de travail mais elles vont de pair avec un certain 
flou sur le plan des services offerts et un accroissement des possibilités de mésententes entre les travailleuses et les clients. Par ailleurs, dans ce contexte, les travailleuses doivent composer avec les tentatives de certains clients de pousser les limites, d'essayer d'obtenir des services non offerts. Ils peuvent même recourir à des menaces de dénonciation si on ne répond pas à leurs exigences. La relation entre la travailleuse et le client est donc toujours potentiellement délicate et peut mettre en péril le travail même de la travailleuse du sexe.

Si les difficultés de protéger les lieux de travail affectent surtout les travailleuses du sexe de rue et celles qui ont opté pour le travail dans des établissements, toutes les travailleuses interviewées, qu'elles soient escortes, travailleuses du sexe de rue ou d'établissements éprouvent des problèmes à être reconnues comme des citoyennes «fiables» et «solvables» dans les échanges économiques. Elles doivent mettre en place des mesures pour éviter le discrédit dans des activités économiques comme la location d'un logement, ou encore l'obtention d'un prêt hypothécaire. Elles doivent penser également à cacher leur insertion dans le travail du sexe pour ne pas forclore leurs possibilités d'entrer sur le marché du travail reconnu.

Considérons d'abord la question du logement. Dans notre recherche sur les travailleuses du sexe de rue de la région d'Ottawa-Gatineau, plusieurs travailleuses ont éprouvé des problèmes pour louer ou encore conserver un appartement. Mais comme plusieurs ont des problèmes de consommation de drogues et peinent à respecter leur bail, il ne nous a pas été possible de distinguer cette dimension du problème des réactions engendrées par leur choix de travail. Quant aux travailleuses dans des établissements, elles gèrent l'information de façon à ne pas être discréditées. Elles ne renseignent le propriétaire sur leur travail que si elles peuvent être confiantes de son ouverture face au travail du sexe. Ainsi, Anaïs a révélé sans problèmes son travail à sa propriétaire, activiste pour la cause homosexuelle et ouverte sur le plan de la morale sexuelle. Par ailleurs, lorsqu'elles ne peuvent parler directement à leur propriétaire, et c'est généralement le cas, elles recourent encore une fois à des stratégies d'évitement qui leur permettent de ne pas révéler cette source de revenus. Ainsi, certaines louent un appartement avec d'autres ou encore le sous-louent et n'ont pas à rendre des comptes de leur travail. D'autres cumulent deux emplois et présentent le second, que ce soit dans le domaine de la restauration, de la décoration, etc. comme leur seul emploi. Certaines, enfin, donnent une fausse information. 
Si l'on examine les demandes de prêts, d'hypothèques aux institutions financières, on constate encore une fois qu'il est très difficile pour les travailleuses du sexe de se présenter comme des citoyennes fiables. Dans notre recherche sur les travailleuses du sexe de rue et sur celles qui travaillent en établissements, les femmes n'ont pas fait état de ce problème. On peut supposer que certaines n'avaient pas suffisamment de revenus pour penser en termes d'emprunts et que d'autres, persuadées qu'elles essuieraient un refus des institutions financières, se sont exclues elles-mêmes. Par contre, des escortes ont fait état de cette difficulté. Marianne, étudiante et escorte, épargnait en vue de l'achat d'une maison et cherchait à se qualifier pour l'obtention d'une hypothèque. Elle s'est constituée en compagnie fictive de services de secrétariat, déclare des revenus et paie des impôts chaque année. Mais pour contourner ce problème, il faut être bien renseignée et bien organisée.

Enfin, comment se présenter sur le marché du travail sans encourir de discrédit et pouvoir réorienter éventuellement sa trajectoire de travail ? Là encore, ce sont les entrevues menées avec des travailleuses escortes qui nous ont fourni des renseignements sur les stratégies possibles. D'abord, nombre d'entre elles poursuivent ou ont poursuivi des études collégiales et universitaires tout en travaillant. Cette mesure évite, d'une part, les trous dans l'histoire d'emploi et peut permettre, d'autre part, d'élargir les options de travail. Certaines exercent plus d'un emploi à la fois, ce qui leur permet de se présenter comme actives sur le marché du travail régulier et d'inscrire dans leur curriculum vitæ d'autres compétences professionnelles reconnues acquises au fil du temps. Par exemple, un travailleur du sexe escorte que nous avons interviewé, était en même temps employé par une compagnie de danse qui donnait une vingtaine de spectacles par session. Il avait aussi suivi des cours de gestion le soir, bénéficié de bourses d'études et occupé d'autres emplois à temps partiel. Aussi son curriculum vitæ était assez solide et ne soulevait pas de doute quant à son insertion dans le marché du travail régulier. Quelques escortes ont aussi fait état de leur participation à l'entreprise familiale. En s'appuyant ainsi sur leur capital social, elles pouvaient maintenir dans l'ombre leur insertion dans le travail du sexe et s'établir professionnellement sans encourir de discrédit. 


\section{Conclusion}

Nos recherches ont mis en évidence que les lois criminelles canadiennes créent des obstacles majeurs à la reconnaissance des travailleuses du sexe comme des travailleuses indépendantes ou des employées malgré leur insertion évidente dans la vie économique. Elles nous ont permis de cerner les barrières de première ligne qui se dressent devant elles, soit celles qui découlent des dispositions des lois criminelles face à la prostitution. Mais comme les travailleuses demeurent fortement en marge de la vie économique, elles ne peuvent prendre acte de leur exclusion en vertu de dispositions relatives à d'autres législations dont le droit du travail. Pivot Legal Society a publié en 2006 un rapport qui prend comme point de départ l'abrogation des lois criminelles sur la «prostitution» et propose d'examiner l'usage potentiel de différentes lois pour assurer dans ce sillage le respect des droits humains des travailleuses du sexe et mettre fin à la violence et à la discrimination dont elles font l'objet. Il examine tour à tour les règlements municipaux, les lois du travail, les règlements relatifs aux prestations sociales et à l'assurance emploi, les lois sur l'impôt des contribuables, les lois de compagnies et propose des changements susceptibles d'assurer l'intégration des travailleuses du sexe comme des travailleuses à part entière. L'examen de chacune de ces lois nous permet de mesurer différentes facettes de la marginalisation économique des travailleuses du sexe. Dans le contexte actuel, elles ne peuvent, par exemple, réclamer des conditions de travail décentes en vertu de la loi sur les conditions de travail, elles ne peuvent avoir accès à des compensations en vertu des lois sur les accidents de travail, elles doivent présenter un rapport d'impôt mais ne peuvent réclamer une déduction pour des frais d'achats de produits nécessaires à leur travail, elles ne peuvent se syndiquer, etc. En somme, elles sont des agentes économiques invisibles et sans droits, largement exploitées par des États qui refusent et profitent tout à la fois de leur contribution à la vie économique de nos sociétés.

\section{Références}

Bauman, Z. (1999). Le cô̂t bumain de la mondialisation. Paris: Hachette.

Bell, L. (1987). Good Girls/Bad Girls. Sex Trade Workers and Feminist Face to Face. Toronto: Women's Press.

Brock, D. (1998). Making work, Making Trouble. Prostitution as a Social Problem. Toronto: University of Toronto Press. 
ConStellation (2005a). Spécial prison, 9, 1, hiver 2005.

ConStellation (2005b). Hors série spécial Stella 10 ans, avril 2005.

Corriveau, P. (2010). Réguler le travail du sexe. Entre la victimisation et la liberté de choisir. In C. Parent, C., Bruckert, P. Corriveau, M. N. Mensah \& L. Toupin (Ed.), Mais Oui c'est un travail! Penser le travail du sexe au-delà de la victimisation (29-54). Montréal: Les Presses de l'Université du Québec.

Dahrendorf, R. (1973). Homo Sociologicus. London: Routledge \& Kegan Paul.

Duchesne, D. (1997). La prostitution de rue au Canada. Juristat, Centre canadien de la Statistique Juridique, Statistique Canada-N ${ }^{0}$ 85-002-XPF, vol 17, no 2.

Foucault, M. (1976). Histoire de la sexualité, 1, La volonté de savoir. Paris: Gallimard.

Gemme, R., \& Payment, N. (1992). Une modification législative au Canada et ses effets sur le phénomène social de la prostitution. In La Prostitution. Quarante ans après la convention de New York (153-185). Actes du Congrès École des sciences criminologiques Léon Cornil et le Centre de rechercheaction et de consultations en sexo-criminologie. Bruxelles: Bruylant. (1, 2, et 3 mars 1990).

Goffman, E. (1963). Stigmates. Les usages sociaux des handicaps. Paris : Les Éditions de Minuit.

Hubbard, P., \& Sanders, T. (2003). Making Space for Sex Work: Female Street Prostitution and the Production of Urban Space. International Journal of Urban and Regional Research, 27 (1), 75-89.

Lowman, J. (2000). Violence and the outlaw status of (street) prostitution. Violence Against Women, 6 (9), 987-1011.

Lowman, J., \& Fraser, L. (1995). Violence against Persons Who Prostitutes: The Experience of British Columbia. Ottawa: Ministère de la Justice.

Mathieu, L. (2001). Mobilisation des prostituées. Paris: Belin.

Mensah, M. N. (2010). L'idée de communauté et l'action collective. In C. Parent, C. Bruckert, P. Corriveau, M. N. Mensah \& L. Toupin (Eds.), Mais Oui c'est un travail! Penser le travail du sexe au-delà de la victimisation (79-106). Montréal: Les Presses de 1'Université du Québec.

Moeller, H.-G. (2006). Lubmann Explained. From Souls to Systems, Chicago and La Salle. Illinois: Open Court.

Parent, C. (2001). Les identités sexuelles et les travailleuses de l'industrie du sexe à l'aube du nouveau millénaire. Sociologie et Sociétés, XXXIII (1), 159-178.

Parent, C., \& Bruckert, C. (2005). Le travail du sexe dans les établissements de services érotiques: une forme de travail marginalisé. Déviance et Société, 29 (1), 33-53.

Parent, C., \& Bruckert, C. (2006). Répondre aux besoins des travailleuses du sexe de rue: un objectif qui passe par la décriminalisation de leurs activités de travail. Reflets, 11, 112-145.

Parent, C., \& Coderre, C. (2000). Le corps social de la prostituée: regards criminologiques. In S. Frigon \& M. Kérésit (Eds.), Du corps des femmes. Contrôles, surveillances et résistances (92-124). Ottawa: Les Presses de l'Université d'Ottawa. 
Pherterson, G. (1986). A Vindication of the Rights of Whores. Seattle, WA: Seal Press.

Pivot Legal Society (2006). Beyond Decriminalisation: Sex Work, Human Rights and a New Framework for Law Reform. Vancouver, B. C. Consulté le 2 août 2010 sur: www.pivotlegal.org/pdfs/Beyond DecrimLongReport.pdf.

Réseau juridique canadien VIH/SIDA (2005). Sex Work, Rights: Reforming Canadian Criminal laws on Prostitution. Toronto. Accessible sur www.aidslaw. ca

Rose, N. (2000). Government and Control. British Journal of Criminology, 40, 321-329.

Shaver, F. M. (1994). The Regulation of Prostitution: Avoiding the Morality Traps. Revue canadienne de droit et société, 9 (1), 123-145.

\begin{abstract}
Starting from the point of departure that sex work is a form of service sector labour, this article explores how sex workers, their own understanding of themselves as workers and consumers notwithsanding, are marginalized in the economic sphere. The article draws attention to the implications of the barriers to their incomegenerating activities (whether they decide to work on the street or not). The article also examines workers resistance and the avoidance strategies they employ attending in particular to how they challenge their marginalization as workers and consumers (obtaining credit cards, mortgages, apartment rentals, etc.). In conclusion, we examine the impact of the laws on the careers of these women noting that while the Criminal Code provisions have a significant impact there are numerous other laws that undermine their ability to define themselves as workers in their own right.
\end{abstract}

KEYWORDS - SeX worker, economic life, marginalization, resistance, avoidance strategies, prostitution.

RESUMEN - A partir de una perspectiva en que el trabajo sexual es visto como una forma de trabajo perteneciente al sector servicios, este artículo explora la manera en que las trabajadoras del sexo, pese a su autodefinición como trabajadoras y consumidoras, son mantenidas al margen de la vida económica. Se describen las diferentes estrategias públicas que afectan sus actividades generadoras de ingresos, tanto si optan por la calle como espacio de trabajo como si no lo hacen. Se exploran también las resistencias y sobre todo las estrategias de que ellas se sirven para contrarrestar dichas tentativas de marginalización en el ámbito de su trabajo y en el del consumo (obtención de tarjetas de crédito, hipotecas, alquiler de apartamento, etc.). En conclusión, se subraya el impacto de las leyes sobre su trayectoria profesional: las disposiciones del Código criminal tienen, en efecto, un impacto mayor sobre ésta, pero un gran número de leyes obstaculizan también sus posibilidades de definirse plenamente como trabajadoras.

PALABRAS CLAVE - Trabajo sexual, vida económica, marginalización, resistencia, prostitución. 